\title{
Impact of self-financed rotavirus vaccines on hospital stays and costs in Spain after a 3-year introductory period
}

\author{
O. REDONDO-GONZÁLEZ ${ }^{1 *}$, J.M. TENÍAS-BURILLO ${ }^{2}$ AND \\ J. RUIZ-GONZALO ${ }^{3}$ \\ ${ }^{1}$ Research Support Unit, La Mancha Centro General Hospital, Alcázar de San Juan, 13600 Ciudad Real, Spain \\ ${ }^{2}$ Preventive Medicine Unit, Pare Jofré Hospital, 46017 Valencia, Spain \\ ${ }^{3}$ Service of Economic Management Control, La Mancha Centro General Hospital, Alcázar de San Juan, 13600 \\ Ciudad Real, Spain
}

Received 30 November 2016; Final revision 26 January 2017; Accepted 2 March 2017;

first published online 3 April 2017

\section{SUMMARY}

Vaccination has reduced rotavirus hospitalizations by $25 \%$ in European regions with low-moderate vaccine availability. We aimed to quantify the reduction in hospital costs after the longest period in which Rotarix ${ }^{\circledR}$ and Rotateq $^{\circledR}$ were simultaneously commercially available in Spain. Cases, length of stay (LOS), and diagnosis-related groups (DRGs) were retrieved from the Minimum Basic Data Set. Healthcare expenditure was estimated through the cost accounting system Gescot ${ }^{\mathbb{B}}$. DRGs were clustered: I, non-bacterial gastroenteritis with complications; II, without complications; III, requiring surgicallother procedures or neonatal cases (highest DRG weights). Comparisons between pre (2003-2005)- and post-vaccine (2007-2009) hospital stays and costs by DRG group were made. Rotaviruses were the most common agents of specific-coded gastroenteritis $(N=1657 / 5012)$. LOS and extended LOS of rotaviruses fell significantly in $2007-2009(\beta$-coefficient $=-0 \cdot 43,95 \%$ confidence intervals $(95 \% \mathrm{CI})-0 \cdot 68$ to $-0 \cdot 17$; and odds ratio $0 \cdot 62,95 \%$ CI $0 \cdot 50-0 \cdot 76$, respectively). Overall, costs attributable to rotavirus hospitalizations fell approximately €244 per patient (95\% CI -365 to -123$)$; the decrease in DRG group III was $€ 2269$ per patient (95\% CI -4098 to -380$)$. We concluded modest savings in hospital costs, largely attributable to cases with higher DRG weights, and a faster recovery. A universal rotavirus vaccination program deserves being re-evaluated, regarding its potential high impact on both at-risk children and societal costs.

Key words: Hospital costs, length of stay, rotavirus, vaccines, Spain.

\section{INTRODUCTION}

Rotavirus is the most frequently identified agent of infectious acute gastroenteritis (AGE) in unvaccinated children under 5 years of age [1-4]. It is associated with significant morbidity and cost across Western

\footnotetext{
* Author for correspondence: O. Redondo-González, MD, Research Support Unit, La Mancha Centro General Hospital, Alcázar de San Juan, 13600 Ciudad Real, Spain.

(Email: oredgon@gmail.com)
}

Europe [5, 6]. In Europe as a whole, the median length of stay (LOS) for rotavirus hospitalizations is $4 \cdot 8$ days (range 2-9.5) [1, 7], 1.4 days longer than the average stay in North America [8]. Moreover, hospitalization charges for rotavirus among European children ranges from $€ 1417 \cdot 44$ to $2263 \cdot 79$ per admission $[9,10]$. For example, the average LOS in Spain is 4.7 days [standard deviation (s.D.) 4.9 days], at an average cost of $€ 1580 \cdot 33$ per admission, which translates to approximately €2.86 million in annual direct costs that are paid 
out by the Spanish National Health System (SNHS) and another $€ 50$ million in estimated societal costs [11]. Compared with healthy children, vulnerable children with co-morbidities have, on average, 8 days greater LOS and six times higher hospital costs $(P<0 \cdot 001)$ [12]. Children aged 6 months and younger are also at increased risk of prolonged LOS from rotavirus [13].

After implementing a rotavirus vaccination program during the period between 2008 and 2009, the reduction in costs associated with hospitalizations in the USA reached US\$ 242 million [14, 15]. Likewise, subsidized vaccination programs in Europe led to an annual decrease of $74 \%$ in the incidence rate of rotavirus hospitalizations after such period, representing a $73 \%$ annual reduction of direct costs [16, 17]. Mean gross cost savings after implementation of rotavirus vaccination are expected to be $€ 304$ per avoided case; and up to $59 \%$ of total savings would be due to herd protection [18]. In Spain, the reduction of the incidence of rotavirus hospitalizations during 20082009 was $33 \cdot 1 \%$ [19], similar to the $25-36 \%$ decrease observed in other European regions with low-tomoderate commercialization [20]. Moreover, nosocomial acute rotavirus gastroenteritis (ARGE) decreased by $37 \cdot 1 \%$ after the period $2007-2009$ [21]. This is consistent with a low-to-moderate rotavirus vaccine coverage in Spain ranging from 17\% in 2007 to $38 \%$ in 2009 [4].

Diagnosis-related groups (DRGs) are indicators of hospital resource consumption and performance. Hospitalized cases classified as belonging to a particular DRG are characterized by a homogenous resource consumption pattern, which involves both direct and indirect hospital costs. Thus, cases within the same DRG are medically and economically similar. DRGs use was approved by the SNHS in 1997 [22]. Still, scientific publications on costs analyses of DRGs in infectious diseases in Europe are scarce despite their growing importance for determining hospital reimbursement [23-28]. Specifically, a national estimate of reduction in costs following the decrease in rotavirus hospitalizations $[4,19]$ has not been estimated to date.

Our aim was to quantify the impact on hospital LOS and costs after the longest time period in which both Rotarix ${ }^{\circledR}$ and Rotateq ${ }^{\circledR}$ were commercially available in Spain.

\section{METHODS}

\section{Design, setting, and data source}

This is a retrospective cohort study of communityacquired ARGE requiring hospital admission in
Castile-La Mancha (CLM), Spain, from 1 January 2003 to 31 December 2009. CLM is a region with a population of over 2 million inhabitants and 19 tertiary referral hospitals. CLM have five major cities (provinces): Guadalajara, Toledo, Ciudad Real, Cuenca, and Albacete. Patients with ARGEs admitted to one privately owned hospital and 12 referral hospitals belonging to the Health Service of Castile-La Mancha (SESCAM), were identified from the Minimum Basic Data Set (MBDS), which uses the codes set forth in the International Classification of Diseases, 9th revision, Clinical Modification (ICD-9-CM).

Altogether, 23006 case records were retrieved: bacterial or parasitic AGEs (001-007, and 008.0-008.5), viral AGEs (008.6-008.8), and AGEs of unknown etiology: 'undetermined cause' (009·0-009·3), 'unspecified non-infectious AGE' (558.9), or 'diarrhea' (787.91), any of which entered into any of the primary or secondary diagnosis fields (SDFs) [4]. The Information and Sanitary Statistics Service of the Counsel for Health and Social Welfare in CLM provided us with the MBDS, specifically including the corresponding DRGs attributable to rotavirus. Weights and costs of DRGs were obtained from the cost accounting system Gescot $^{\circledR}$, run by the Economic Management Control Office of the General Secretary of SESCAM.

\section{Definition of variables}

Specified AGEs were defined by the presence of a specific ICD-9-CM code of bacterial, parasitic, or viral AGE in the primary diagnosis field of the MBDS, as well as by any of these codes in any SDFs accompanied by a principal diagnosis code of unknown etiology. In particular, rotaviruses ('008.61') and the other most commonly coded cause-specific AGEs were recovered this way (Salmonella '003.0-003.9,' Campylobacter '008.43,' adenovirus '008.62', and other viral enteritis ' $008 \cdot 8$ '). Non-specified (or non-typified) AGEs were those with a primary diagnosis code of unknown AGE and no specific code in their corresponding SDFs [4].

LOS (in days) was estimated from the hospital admission and discharge dates. Extended LOS was defined $a d$ hoc as any hospital stay over 3 days, as that was the median LOS for rotaviruses in CLM during the study period. Hospital readmission due to "the same process' was defined as any hospitalization of the same patient twice in a 1-month period.

Medical costs were estimated as mean costs per DRG for each hospital and year of admission, in 
accordance with the Spanish norms that were operative throughout the study period (All Patient-DRG Groups, versions 18 and 23). These data are given in Supplementary Table S1. For the purposes of description and analysis, DRGs were clustered in three main groups: I. Non-bacterial AGE with complications (DRGs 813, 815); II. Non-bacterial AGE without complications (DRGs 777, 814, 816); and III. Highly weighted DRGs: admissions requiring surgical or other procedures as well as newborns requiring admission (DRGs 156, 468, 551, 613, 627, 628). Additional information on SESCAM's casuistic and functional indices can be found in Supplementary Table S2.

The 2007-2009 period was defined as the vaccine triennium, as it was the introductory and the longest time period in which both vaccines were commercially available in Spain simultaneously. For comparison purposes, the period between 2003 and 2005 was defined as the pre-vaccine period. Because only Rotarix ${ }^{\circledR}$ was made commercially available in Spain starting in July, 2006, that year was considered to be a transition period and was not included in our study.

Epidemiological factors, comorbidities and complications accompanying rotavirus-coded hospitalizations were retrieved from the SDFs and analyzed elsewhere [29].

\section{Statistical analysis}

First, we categorized the total number of bed days corresponding to coded AGEs, both specific and nonspecific, throughout the study period by age groups ( $<5$ years or 5 years and up). For LOS, the mean and the S.D. were used. For extended LOS, absolute and relative frequencies were employed. For intra-group age $(<5$ or $\geqslant 5$, and total), comparisons of LOS and extended LOS between the main types of AGEs were calculated with the aid of the Student's $t$ test and the $\chi^{2}$ test, respectively. Pre- and post-vaccine LOS and extended LOS were described also separated into the two age groups, given the reported differences in prevalence of the different pathogens causing AGE that exist according to age [14]. Then, an adjusted analysis by age both of LOS and extended LOS was carried out for the most commonly coded community-acquired infectious AGEs requiring hospitalization (apart from rotaviruses) between the pre- and post-vaccine periods.

Afterwards, two estimate model approaches were specifically built for the stays of rotavirus-coded hospitalizations, simultaneously adjusting for those confounding or interacting (modifying) features that were identified in a previous stratified analysis of LOS and extended LOS between both 3-year periods: a multivariate linear regression analysis for LOS and a multivariate logistic regression analysis for extended LOS.

To quantify the magnitude of the association both for adjusted analysis by age of the most commonly coded AGEs and for multivariate regression analysis, we estimated the $\beta$-regression coefficient in the case of LOS and the odds ratio (OR) for extended LOS, both with their 95\% confidence intervals $(95 \% \mathrm{CI})$.

Additionally, a disaggregated analysis was made to explore the potential effect of vaccination on the decrease in LOS, both for hospitalized patients with rotavirus who presented comorbidities (perinatal problems, weight-height disorders, or co-infections), and for the most severe cases that needed intravenous rehydration.

Finally, analysis of the costs associated with DRGs attributable to rotavirus-coded hospitalizations in 2007-2009 were compared with those in 2003-2005. Prior to this, we corrected the annual average costs represented by each DRG in accordance with the Consumer Price Index for each year, obtained from an online application of the Spanish National Institute of Statistics. For each DRG group in each 3 -year period, quality variables (readmission rates) and efficacy variables (mean hospital stay and DRG-based costs) were calculated. Pre-post comparisons of costs and stays by DRG group were analyzed with the independent Student's $t$ test; the $\chi^{2}$ test was used to compare readmission rates.

All analyses were performed using Stata 12 Statistical Software (StataCorp LP, TX); the level of significance $(P)$ was assigned to values $<0 \cdot 05$.

\section{Ethics}

The Ethical Review Board of the Service of Information and Sanitary Statistics of the Counseling of Health and Social Welfare in CLM approved this study.

\section{RESULTS}

A total of 17415 AGEs were recovered throughout the study period; 5012 (29\%) were coded as causespecific. The most frequently coded agents were rotavirus $(N=1657 ; 33 \%)$, Salmonella spp. $(N=1581$; $32 \%)$, undefined viral agents $(N=446 ; 9 \%)$, 
Campylobacter $(N=337 ; 7 \%)$, and adenovirus $(N=$ $120 ; 2 \%)$.

Between 2003 and 2009, the average stay for ARGEs requiring hospitalization in children under 5 year was $3.79 \pm 4.04$ days; $45 \%$ of these ARGEs had an extended LOS (Table 1). Figure 1 shows the mean distribution of LOS further broken down into different ages. In the case of rotavirus, the longest average hospital stay was found in children under the age of 7 months ( $4 \cdot 51$ days, 95\% CI $4 \cdot 17-4 \cdot 85$ ). In the case of other specified AGEs as well as nonspecified AGEs, the longest mean LOS was for children $\geqslant 10$ years (6.76 days, 95\% CI 6.40-7.06 and 4.96 days, 95\% CI $4 \cdot 81-5 \cdot 11$, respectively). The mean LOS of patients with perinatal problems affected by ARGE was 3.34 days greater than for those without $(95 \%$ CI $2 \cdot 54-4 \cdot 14, \quad P<0.0001)$. Similarly, LOS of patients with weight-height developmental disorders or with at least one concomitant infection in addition to ARGE was 1.12 and 1.00 days greater than for those without these additional problems $(95 \%$ CI $0 \cdot 49-1 \cdot 74, P=0.0005)$ and $(95 \%$ CI $0 \cdot 70-1 \cdot 29, P<0 \cdot 0001)$, respectively.

Figure 2 represents annual distribution of LOS and extended LOS for the main groups of gastroenteritis overall, according to their codification: a general downward trend of LOS during the vaccine period can be observed in all cases, even in non-specified AGEs. However, as shown in Table 2, a significant decline in the mean LOS was only found for rotavirus ( $\beta$-coef. $=-0.43,95 \% \quad \mathrm{CI}-0.68$ to $-0 \cdot 17)$ and Campylobacter $(-1 \cdot 35,95 \%$ CI -2.39 to -0.30$)$. Unlike rotaviruses, LOS of hospitalizations due to Campylobacter was $4 \cdot 22$ days longer in patients $\geqslant 5$ years $(95 \%$ CI $3 \cdot 15-5 \cdot 28, P<0 \cdot 0001)$. With respect to other non-specified AGEs, LOS experienced a non-significant decrease in children $<5$ years $(-0.07,95 \%$ CI -0.43 to $0.28, P=0.693)$ during the vaccine period, but increased in children $\geqslant 5$ years of age $(0 \cdot 44 ; 95 \%$ CI $0 \cdot 17-0 \cdot 70 ; P=0 \cdot 001)$. Extended LOS also decreased significantly in the case of rotavirus (OR $0 \cdot 62,95 \%$ CI $0 \cdot 50-0 \cdot 76$ ) and Campylobacter (OR 0.32 , 95\% CI $0.19-0.55$ ) (Table 3). Again, the mean extended LOS for Campylobacter was four times higher in patients $\geqslant 5$ year $(95 \%$ CI $2 \cdot 42-7 \cdot 49, P<0 \cdot 0001)$. During the vaccine period, extended LOS for 'other nonspecified AGEs' decreased significantly in children $<5$ year (OR 0.840; 95\% CI 0.736-0.958, $P=$ $0 \cdot 010$ ), but increased non-significantly in those $\geqslant 5$ (OR $1 \cdot 05$; 95\% CI $0 \cdot 96-1 \cdot 15, P=0 \cdot 254$ ).
After a stratified analysis of decrease in LOS for rotavirus hospitalizations, no interacting variables were found, and only 'provinces of residence' turned out to be a confounding variable in such decrease: crude $\beta$-coef. $=-0.42(95 \% \mathrm{CI}-0.68$ to $-0 \cdot 17, P=$ $0 \cdot 001)$; and adjusted $\beta$-coef. by provinces of residence $=-0 \cdot 39,(95 \% \mathrm{CI}-0.64$ to $-0 \cdot 14, P=0 \cdot 002)$. The consequent multivariate linear regression analysis including 'provinces of residence' showed that the decrease in LOS was only significant in Ciudad Real province $\quad(\beta$-coef. $=-0.31, \quad 95 \% \quad \mathrm{CI} \quad-0.609$ to $-0 \cdot 140, \quad P=0.040)$ and Guadalajara province $(\beta$-coef. $=-0.99, \quad 95 \% \quad$ CI -1.56 to $-0.43, \quad P=$ $0 \cdot 001)$. In the stratified analysis for extended LOS, we did not find any variable that confounded or modified the decrease of extended LOS in the vaccination period.

We found non-significant decrease in the LOS for rotavirus hospitalizations with concomitant perinatal problems $(P=0.135)$ or weight-height disorders $(P$ $=0.822)$ in 2007-2009; but there was a significant decrease in LOS of ARGEs with at least one concomitant infection $(\beta$-coef. $=-0.66,95 \%$ CI -1.25 to $-0.075, P=0.027)$ and in those requiring intravenous rehydration $(\beta$-coef. $=-1 \cdot 37,95 \% \mathrm{CI}-2 \cdot 56$ to $-0 \cdot 17$, $P=0 \cdot 025)$.

Global costs attributable to rotavirus hospitalizations went down approximately $€ 243.65$ per patient (95\% CI $-365 \cdot 18$ to $-123 \cdot 18, P=0 \cdot 0001$ ) during the vaccine period, with a $1.5 \%$ decrease $(95 \% \mathrm{CI}-2 \cdot 8$ to $-0.2 \%)$ in the readmission rate $(P=0.0318)$ (Table 4). A real decrease of $€ 2268.83$ per patient was observed for more highly weighted DRGs $(95 \%$ CI -4097.87 to $-379.76 ; \quad P=0.0204)$, since the mean cost for DRGs corresponding to groups I and II rose by $€ 476.79$ per patient in 2007-2009 (95\% CI 408.98 to $544.61 ; P \leqslant 0 \cdot 001)$. Besides, the average decrease in LOS for DRGs owing to group III was 3.56 days $(95 \% \mathrm{CI}-5 \cdot 28$ to $-1 \cdot 84 ; P<0 \cdot 0001)$.

\section{DISCUSSION}

After the 2007-2009 period with a mean vaccine coverage of $34 \%$ in CLM (from $18 \%$ in 2007 to $44 \%$ in 2009), we found a $14 \%$ decrease in the median rate of rotavirus hospitalizations $(95 \%$ CI 4-22) [4] leading to a modest overall savings of $€ 26071$ for SESCAM, which were largely attributable to a fall in cases with higher DRG weights (representing nearly $€ 2300$ per patient). While the overall mean LOS of rotavirus hospitalizations fell by almost half a day (a 
Table 1. Comparison of LOS for all (specific and non-specific) coded AGEs in the 2003-2009 period by age groups (Source: MBDS, Castile-La Mancha, Spain*)

\begin{tabular}{|c|c|c|c|c|}
\hline LOS & ARGE & $\begin{array}{l}\text { Other specified } \\
\text { AGEs }\end{array}$ & $\begin{array}{l}\text { Non-specified } \\
\text { AGEs }\end{array}$ & $P$-value \\
\hline \multicolumn{5}{|l|}{$<5$ year } \\
\hline $\mathrm{N}$ admissions (\%) & $1623(98 \%)$ & $1059(32 \%)$ & $4507(36 \%)$ & \\
\hline Mean \pm S.D. & $3 \cdot 79 \pm 2 \cdot 35$ & $4 \cdot 15 \pm 2 \cdot 61$ & $3 \pm 2 \cdot 10$ & $0 \cdot 001$ \\
\hline Range & $(0-31)$ & $(0-124)$ & $(0-57)$ & \\
\hline Median (IR) & $3(2-5)$ & $4(2-5)$ & $3(2-4)$ & \\
\hline Extended LOS N (\%) & $737(45 \%)$ & $536(51 \%)$ & $1321(29 \%)$ & $0 \cdot 001$ \\
\hline \multicolumn{5}{|l|}{$\geqslant 5$ year } \\
\hline $\mathrm{N}$ admissions (\%) & $34(2 \%)$ & $2296(68 \%)$ & $7895(64 \%)$ & \\
\hline Mean \pm S.D. & $3 \cdot 79 \pm 4 \cdot 04$ & $6 \cdot 76 \pm 7 \cdot 42$ & $4 \cdot 97 \pm 6 \cdot 91$ & $<0 \cdot 0001$ \\
\hline Range & $(0-23)$ & $(0-159)$ & $(0-357)$ & \\
\hline Median (IR) & $3(2-4)$ & $5(3-8)$ & $3(2-6)$ & \\
\hline Extended LOS N (\%) & $11(32 \%)$ & $1543(67 \%)$ & $3822(48 \%)$ & $<0 \cdot 0001$ \\
\hline \multicolumn{5}{|l|}{ Total } \\
\hline $\mathrm{N}$ admissions (\%) & $1657(100 \%)$ & $3355(100 \%)$ & $12403(100 \%)$ & \\
\hline Mean \pm S.D. & $3 \cdot 79 \pm 2 \cdot 40$ & $5 \cdot 21 \pm 5 \cdot 51$ & $4 \cdot 26 \pm 5 \cdot 74$ & $<0 \cdot 0001$ \\
\hline Range & $(0-31)$ & $(0-159)$ & $(0-357)$ & \\
\hline Median (IR) & $3(2-5)$ & $4(3-6)$ & $3(2-5)$ & \\
\hline Extended LOS N (\%) & $748(45 \%)$ & $2079(62 \%)$ & $5143(41 \%)$ & $<0 \cdot 0001$ \\
\hline
\end{tabular}

IR, interquartile range.

* The assumption of normality by age group for the three types of AGEs was confirmed with the aid of the Shapiro-Wilks test.

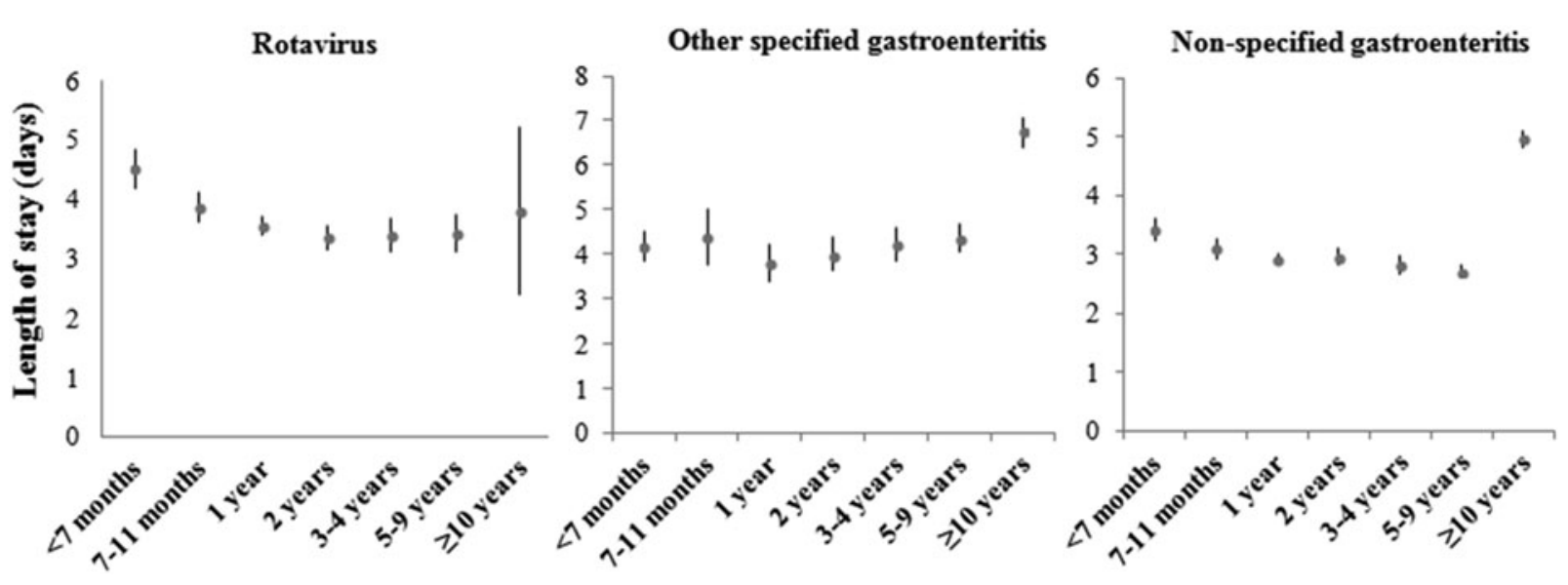

Fig. 1. Distribution of means for LOS (in days) with their 95\% CI, by age groups, for rotavirus and other specific and non-specific coded AGEs. Period 2003-2009. Source: MBDS of CLM, Spain.

$10 \%$ decrease); the mean LOS for the highest DRGs fell by almost 4 days (an eightfold higher decrease). Further, the decrease in LOS was $7 \%$ and $24 \%$ greater for ARGEs accompanied by at least one concomitant infection or requiring intravenous rehydration, respectively; and extended LOS fell by $38 \%$.

A prospective observational study conducted from October 2008 to June 2009 covering the regions of North-west Spain concluded that indirect societal costs due to ARGE in children up to 5 years old ( 192.7€ per family) were $1 \cdot 74$-fold higher compared with AGEs of other etiologies [30]. Although the aim of our work was not determining societal costs, the projection of such value to the 709 hospital admissions avoided during the vaccination period would allow us to estimate an approximate additional saving of $€ 136624$; of which, $€ 85364$ would be due to absenteeism. 


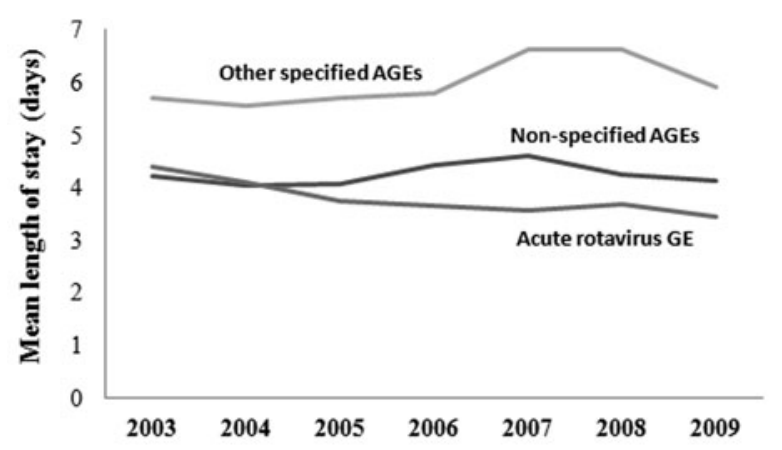

also emphasized that underestimation of ARGE hospitalizations may be also due to the fact that estimations are usually made using only the principal diagnosis [32]; but we have only estimated 17 cases with a primary diagnosis code of non-typified AGE and a specific code of rotavirus in their corresponding SDFs [4]. Anyway, poor classification of non-specified gastroenteritis events (including rotavirus cases in this group) can only lead to a dilution of the potential impact of the rotavirus vaccine both in terms of costs and stays (bias toward null value or nondifferential classification bias).

In this study we analyzed the decrease of stays apart from that of costs because in SESCAM the price of each DRG is not calculated by the cost attributable to each patient individually, but by multiplying each DRG weight by the 'weight unit' cost (or Hospital Complexity Unit (HCU) cost) owing to the hospitalization 'Homogeneous Functional Group' (HFG) of a department: $\mathrm{HCU}$ cost of a department $=$ total cost of the hospitalization HFG of the department/ total weight of the hospitalization HFG of the department. The calculation of the total weight of the hospitalization HFG is made independently of the stays as $\Sigma$ (unit weight of each DRG $\times$ number of hospital discharges with that DRG); as the hospitalization department would maintain, with rare exceptions, a full stay throughout the year for whatever reason of admission. Therefore, according to this management system, the average stay would not influence directly the HCU cost of a department. Although LOS does not directly influence the costs, the LOS decrease would have an impact on social costs by reducing parental absenteeism, while improving the efficiency of the neonatal-pediatric department, allowing other patients to be hospitalized and thus decreasing the waiting lists.

The decrease in LOS for ARGEs was only significant in Ciudad Real and Guadalajara provinces. The vaccination coverage of both were the largest and the ones that increased the most during the triennium of vaccination, together with Albacete, although here the decrease in LOS did not become significant (Supplementary Table S4). One reason why it did not happen in Albacete could be a misclassification in exposure variable (data provided by laboratories come from distributed, but not administered vaccines, so we do not know to what extent immunization schedules were administered completely); and on the other hand, that hospital stays depend on the own department and/or intra-hospital bed management and its consequent efficiency. 
Table 2. Adjusted analysis of LOS (in days) by age groups for the most common codified community-acquired infectious AGEs requiring hospitalization between the pre (2003-2005)- and post-vaccine (2007-2009) periods in CLM, Spain*

\begin{tabular}{|c|c|c|c|c|c|c|c|}
\hline \multirow[b]{2}{*}{ Hospitalization days $(\mathrm{N})$} & \multicolumn{3}{|c|}{ Pre-vaccine period (2003-2005) } & \multicolumn{3}{|c|}{ Post-vaccine period (2007-2009) } & \multirow{2}{*}{$P$-value } \\
\hline & All & $<5$ years & $\geqslant 5$ years & All & $<5$ years & $\geqslant 5$ years & \\
\hline Rotavirus (N) & 3077 & 3034 & 43 & 2368 & 2312 & 56 & \\
\hline Mean \pm S.D., range & $4 \cdot 01 \pm 2 \cdot 64,0-31$ & $4 \cdot 00 \pm 2 \cdot 64,0-31$ & $3 \cdot 91 \pm 2 \cdot 70,1-10$ & $3 \cdot 58 \pm 2 \cdot 16,0-23$ & $3 \cdot 57 \pm 2 \cdot 03,0-16$ & $4 \cdot 00 \pm 5 \cdot 68,1-23$ & \\
\hline Age-adjusted $\beta$-coef. $\dagger(95 \% \mathrm{CI})$ & $0 \cdot 000$ & & & $-0.43(-0.68$ to -0.17$)$ & & & $0 \cdot 001$ \\
\hline Salmonella $\operatorname{spp}(\mathrm{N})$ & 5421 & 1218 & 4203 & 2690 & 545 & 2145 & \\
\hline Mean \pm s.D., range & $5 \cdot 84 \pm 4 \cdot 31,0-45$ & $5 \cdot 03 \pm 2 \cdot 44,1-16$ & $6 \cdot 12 \pm 4 \cdot 76,0-45$ & $6 \cdot 26 \pm 8 \cdot 541-16$ & $4.95 \pm 2 \cdot 73,1-20$ & $6 \cdot 70 \pm 9 \cdot 73,1-159$ & \\
\hline Age-adjusted $\beta$-coef. $\uparrow(95 \% \mathrm{CI})$ & $0 \cdot 000$ & & & $0 \cdot 41(-0 \cdot 27,1 \cdot 10)$ & & & $0 \cdot 233$ \\
\hline Campylobacter $(\mathrm{N})$ & 815 & 414 & 401 & 720 & 224 & 496 & \\
\hline Mean \pm S.D., range & $5 \cdot 74 \pm 4 \cdot 38,0-24$ & $4 \cdot 27 \pm 2 \cdot 59,0-17$ & $8 \cdot 91 \pm 5 \cdot 63,0-24$ & $5 \cdot 18 \pm 5 \cdot 25,0-38$ & $3 \cdot 25 \pm 2 \cdot 00,0-12$ & $7 \cdot 09 \pm 6 \cdot 61,1-38$ & \\
\hline Age-adjusted $\beta$-coef. $\dagger(95 \% \mathrm{CI})$ & $0 \cdot 000$ & & & $-1.35(-2.39 \text { to }-0 \cdot 30)^{1}$ & & & $0 \cdot 012$ \\
\hline Adenovirus $(\mathrm{N})$ & 112 & 111 & 1 & 186 & 186 & 0 & \\
\hline Mean \pm S.D., range & $3 \cdot 39 \pm 2 \cdot 54,1-12$ & $3 \cdot 47 \pm 2 \cdot 54,1-12$ & - & $3 \cdot 21 \pm 1 \cdot 35,1-7$ & $3 \cdot 21 \pm 1 \cdot 35,1-7$ & - & \\
\hline Age-adjusted $\beta$-coef. $\dagger(95 \% \mathrm{CI})$ & 0.000 & & & $-0.262(-1.075$ to 0.551$)$ & & & $0 \cdot 524$ \\
\hline Undefined viral enteritis ${ }^{\S}(\mathrm{N})$ & 715 & 324 & 391 & 699 & 239 & 460 & \\
\hline Mean \pm S.D., range & $3 \cdot 95 \pm 4 \cdot 74,0-55$ & $3 \cdot 21 \pm 2 \cdot 33,0-16$ & $4 \cdot 89 \pm 6 \cdot 54,1-55$ & $3 \cdot 76 \pm 3 \cdot 04,0-21$ & $2 \cdot 95 \pm 1 \cdot 56,1-10$ & $4 \cdot 38 \pm 3 \cdot 70,0-21$ & \\
\hline Age-adjusted $\beta$-coef. $\uparrow(95 \% \mathrm{CI})$ & $0 \cdot 000$ & & & $-0 \cdot 383(-1 \cdot 189$ to 0.424$)$ & & & $0 \cdot 352$ \\
\hline Other AGEs $(\mathrm{N})$ & 14137 & 950 & 13187 & 16322 & 950 & 15372 & \\
\hline Mean \pm S.D., range & $4 \cdot 22 \pm 4 \cdot 620-123$ & $3 \cdot 10 \pm 1 \cdot 94,0-18$ & $4 \cdot 93 \pm 5 \cdot 58,0-123$ & $4 \cdot 60 \pm 6 \cdot 90,0-357$ & $3 \cdot 03 \pm 2 \cdot 48,0-57$ & $5 \cdot 36 \pm 8 \cdot 22,0-357$ & \\
\hline Age-adjusted $\beta$-coef. $\dagger(95 \% \mathrm{CI})$ & $0 \cdot 000$ & & & $0.254(0.043-0.466)^{+}$ & & & $0 \cdot 019$ \\
\hline
\end{tabular}

* No significant interaction of 'age group' $(\geqslant 5 /<5$ years) was found in the association between LOS and 3-year period in known AGEs ( $\mathrm{p}$-rotavirus $=0 \cdot 595$; $\mathrm{p}$-salmonella $=$ $0.404 ; \mathrm{p}$-campylobacter $=0.460 ; \mathrm{p}$-adenovirus $=$ non-evaluatable; $\mathrm{p}$-undefined viral enteritis $=0.762)$; but an interaction was found in the case of unknown AGEs $(\mathrm{p}$-other AGEs $=0 \cdot 025)$.

$\dagger \beta$ regression coefficient adjusted by age group ( $\geqslant 5 /<5$ years), comparing LOS (in days) between post- and pre-vaccine periods.

\$ Age was a confounding factor for the association between LOS and the two 3-year periods: LOS of Campylobacter $(\beta$-coef. $\geqslant 5 /<5=4 \cdot 22 ; 95 \%$ CI $3 \cdot 15-5 \cdot 28 ; P<0 \cdot 0001) ;$ LOS of adenovirus $(\beta$-coef. $\geqslant 5 /<5=$ non-evaluatable); LOS of other viral enteritis $(\beta$-coef. $\geqslant 5 /<5=1 \cdot 91 ; 95 \%$ CI $0 \cdot 35-10 \cdot 54 ; P=0 \cdot 457)$.

$\S$ Undefined viral enteritis: ICD-9-CM code ' $008 \cdot 8$ '

I Ninety-eight percent of other AGEs were coded as non-specified (or non-typified) AGEs in the MBDS. 
Table 3. Adjusted analysis (by age group) of extended LOS for the most commonly coded community-acquired infectious AGE requiring hospitalization between the pre (2003-2005)- and post-vaccine (2007-2009) periods in CLM, Spain*

\begin{tabular}{|c|c|c|c|c|c|c|c|}
\hline \multirow[b]{2}{*}{ Hospitalization episodes (N) } & \multicolumn{3}{|c|}{ Pre-vaccine period (2003-2005) } & \multicolumn{3}{|c|}{ Post-vaccine period (2007-2009) } & \multirow{2}{*}{$\begin{array}{c}P \text {-value } \\
\text { (crude/adjusted) }\end{array}$} \\
\hline & All & $<5$ years & $\geqslant 5$ years & All & $<5$ years & $\geqslant 5$ years & \\
\hline Rotavirus (N) & 769 & $758(98 \cdot 6 \%)$ & $11(1 \cdot 4 \%)$ & 662 & $648(97 \cdot 9 \%)$ & $14(2 \cdot 1 \%)$ & \\
\hline $0-3$ days & $379(49 \cdot 3 \%)$ & 372 & 7 & $405(61 \cdot 2 \%)$ & 394 & 11 & \\
\hline$>3$ days (extended stay) & $390(50 \cdot 7 \%)$ & 386 & 4 & $257(38 \cdot 8 \%)$ & 254 & 3 & \\
\hline Age-adjusted $\mathrm{OR} \dagger(95 \% \mathrm{CI})$ & $1 \cdot 00$ & & & $0.62(0.50-0 \cdot 76)$ & & & $<0 \cdot 0001$ \\
\hline Salmonella spp (N) & 929 & $242(26 \cdot 1 \%)$ & $687(74 \cdot 9 \%)$ & 430 & $110(25 \cdot 6 \%)$ & $320(74 \cdot 4 \%)$ & \\
\hline $0-3$ days & $251(18 \cdot 5 \%)$ & 71 & 180 & $126(9 \cdot 3 \%)$ & 37 & 89 & \\
\hline$>3$ days (extended stay) & $678(49 \cdot 9 \%)$ & 171 & 507 & $304(22 \cdot 4 \%)$ & 73 & 231 & \\
\hline Age-adjusted $\mathrm{OR} \uparrow(95 \% \mathrm{CI})$ & $1 \cdot 00$ & & & $0.89(0.69-1 \cdot 15)$ & & & $0 \cdot 378$ \\
\hline Campylobacter $(\mathrm{N})$ & 142 & $97(68 \cdot 3 \%)$ & $45(31 \cdot 7 \%)$ & 139 & $69(49 \cdot 6 \%)$ & $70(50 \cdot 4 \%)$ & \\
\hline $0-3$ days & $46(16 \cdot 4 \%)$ & 41 & 5 & $71(25 \cdot 27 \%)$ & 46 & 25 & \\
\hline$>3$ days (extended stay) & $96(34 \cdot 2 \%)$ & 56 & 40 & $68(24 \cdot 20 \%)$ & 23 & 45 & \\
\hline Age-adjusted $\mathrm{OR} \uparrow(95 \% \mathrm{CI})$ & $1 \cdot 00$ & & & $0 \cdot 32(0 \cdot 19-0.55)^{+}$ & & & $<0 \cdot 0001$ \\
\hline Adenovirus $(\mathrm{N})$ & 33 & $32(96 \cdot 97 \%)$ & $1(3 \cdot 03 \%)$ & 58 & $58(100 \%)$ & 0 & \\
\hline 0-3 days & $24(72 \cdot 7 \%)$ & 23 & 1 & $39(67 \cdot 24 \%)$ & 39 & 0 & \\
\hline$>3$ days (extended stay) & $9(27 \cdot 3 \%)$ & 9 & 0 & $19(32 \cdot 76 \%)$ & 19 & 0 & \\
\hline Age-adjusted $\mathrm{OR} \uparrow(95 \% \mathrm{CI})$ & $1 \cdot 00$ & & & $1 \cdot 25(0 \cdot 48-3 \cdot 21)$ & & & $0 \cdot 650$ \\
\hline Undefined viral enteritis§ (N) & 180 & $101(56 \cdot 1 \%)$ & $79(43.9 \%)$ & 188 & $81(43 \cdot 1 \%)$ & $107(56 \cdot 9 \%)$ & \\
\hline $0-3$ days & $108(60 \%)$ & 66 & 42 & $117(62 \cdot 2 \%)$ & 59 & 58 & \\
\hline$>3$ days (extended stay) & $72(40 \%)$ & 35 & 37 & $71(37 \cdot 8 \%)$ & 22 & 49 & \\
\hline Age-adjusted $\mathrm{OR} \uparrow(95 \% \mathrm{CI})$ & $1 \cdot 00$ & & & $0 \cdot 86(0 \cdot 56-1 \cdot 32)$ & & & $0 \cdot 488$ \\
\hline Other AGEs $(\mathrm{N})$ & 5586 & $2114(37 \cdot 8 \%)$ & $3472(62 \cdot 2 \%)$ & 5758 & $1823(31 \cdot 7 \%)$ & $3935(68 \cdot 3 \%)$ & \\
\hline 0-3 days & $3218(57 \cdot 6 \%)$ & 1434 & 1784 & $3272(56 \cdot 8 \%)$ & 1304 & 1968 & \\
\hline$>3$ days (extended stay) & $2368(42 \cdot 4 \%)$ & 680 & 1688 & $2486(43 \cdot 2 \%)$ & 519 & 1967 & \\
\hline Age-adjusted $\mathrm{OR} \uparrow(95 \% \mathrm{CI})$ & $1 \cdot 00$ & & & $0.98(0.91-1.06)$ & & & $0 \cdot 603$ \\
\hline
\end{tabular}

$\mathrm{N}$, number of hospital admissions (hospital episodes).

* No significant interaction of 'age group' ( $\geqslant 5 /<5$ years) was found in the association between extended LOS and 3-year period in known AGEs ( $p$-rotavirus $=0 \cdot 772 ;$-salmonella $=0.684 ; \mathrm{p}$-campylobacter $=0.438 ; \mathrm{p}$-adenovirus $=$ non-evaluatable; $\mathrm{p}$-undefined viral enteritis $=0.403)$; but an interaction was found in the case of unknown AGEs (p-other AGEs =0.005).

$\dagger$ OR adjusted by age group ( $\geqslant 5 /<5$ years), comparing extended LOS ( $>3$ days) between post- and pre-vaccine periods.

+. Age was a confounding factor for the association between extended LOS of Campylobacter and the two 3 -year periods: OR $\geqslant 5 /<5=4 \cdot 40 ; 95 \%$ CI $2 \cdot 42-7 \cdot 49 ; P<0 \cdot 0001$.

$\S$ Undefined viral enteritis: ICD-9-CM code '008.8'.

TNinety-eight percent of other AGEs were coded as non-specified (or non-typified) AGEs in the MBDS. 
Table 4. Evaluation of change in mean LOS and costs attributable to hospital admissions caused by rotavirus in CLM, Spain (Sources: MBDS, 2003-2009; Cost Accounting System Gescot ${ }^{\circledR}$, Economic Management Control, General Secretary of the Health Service of Castile-La Mancha*)

\begin{tabular}{|c|c|c|c|c|c|}
\hline Characteristics* & DRGs & $\begin{array}{l}\text { Pre-vaccine period } \\
(2003-2005) N=769\end{array}$ & $\begin{array}{l}\text { Post-vaccine period } \\
(2007-2009) N=662\end{array}$ & $\begin{array}{l}\text { Impact } \\
\text { (post-pre change, } 95 \% \mathrm{CI} \text { ) }\end{array}$ & $P$-value \\
\hline Group I (non-bacterial AGE with complications) & 813,815 & $N=145(18 \cdot 86 \%)$ & $N=160(24 \cdot 02 \%)$ & & \\
\hline Mean stay, S.D. (days) & & $3 \cdot 99 \pm 2 \cdot 01$ & $3 \cdot 86 \pm 2 \cdot 17$ & $-0 \cdot 13(-0 \cdot 37$ to $0 \cdot 11)$ & $0 \cdot 0624$ \\
\hline Readmission rate & & $3(2 \cdot 1 \%)$ & 0 & $-2 \cdot 1 \%(-5$ to $0 \cdot 9 \%)$ & $0 \cdot 2143$ \\
\hline Mean cost per patient $(€)$ & & $1428 \cdot 72 \pm 134 \cdot 49$ & $2061 \cdot 77 \pm 164 \cdot 61$ & $633 \cdot 05(599 \cdot 24$ to $666 \cdot 86)$ & $<0 \cdot 0001$ \\
\hline Group II (non-bacterial AGE without complications) & $777,814,816$ & $N=604(78 \cdot 41 \%)$ & $N=481(72 \cdot 51 \%)$ & & \\
\hline Mean stay (days) & & $3 \cdot 81 \pm 1 \cdot 90(2300 / 603)$ & $3 \cdot 35 \pm 1 \cdot 76(1608 / 480)$ & $-0 \cdot 46(-0 \cdot 58$ to $-0 \cdot 34)$ & $0 \cdot 0009$ \\
\hline Readmission rate & & $12(2 \cdot 0 \%)$ & $4(0 \cdot 8 \%)$ & $-1 \cdot 2 \%(-2 \cdot 7$ to $0 \cdot 4 \%)$ & $0 \cdot 1889$ \\
\hline Mean cost per patient $(€)$ & & $1025 \cdot 78 \pm 59 \cdot 97$ & $1346 \cdot 31 \pm 126 \cdot 32$ & $320 \cdot 53(218 \cdot 72$ to $422 \cdot 35)$ & $<0 \cdot 0001$ \\
\hline $\begin{array}{l}\text { Group III (higher DRGs weights: requirements of } \\
\text { surgical/other procedures and/or neonatal cases) } \dagger\end{array}$ & $\begin{array}{r}156,468,551 \\
613,627,628\end{array}$ & $N=20(2 \cdot 73 \%)$ & $N=21(3 \cdot 47 \%)$ & & \\
\hline Mean stay (days) & & $9 \cdot 95 \pm 9 \cdot 55$ & $6 \cdot 39 \pm 5 \cdot 43$ & $-3 \cdot 56(-5 \cdot 28$ to $-1 \cdot 84)$ & $<0 \cdot 0001$ \\
\hline Readmission rate & & $1(4 \cdot 8 \%)$ & 0 & $-4 \cdot 8 \%(-18 \cdot 4$ to $8 \cdot 9 \%)$ & $0 \cdot 9633$ \\
\hline Mean cost per patient $(€)$ & & $4565 \cdot 81 \pm 3776 \cdot 44$ & $2326 \cdot 98 \pm 1409 \cdot 14$ & $-2268 \cdot 83(-4097 \cdot 87$ to $-379 \cdot 76)$ & $0 \cdot 0204$ \\
\hline Groups I, II y III & & $N=769(100 \%)$ & $N=662(100 \%)$ & & \\
\hline Mean stay (days) & & $4 \cdot 01 \pm 2 \cdot 64$ & $3 \cdot 58 \pm 2$ & $-0.43(-0.67$ to $-0 \cdot 19)$ & $0 \cdot 0005$ \\
\hline Readmission rate & & $16(2 \cdot 08 \%)$ & $4(0 \cdot 60 \%)$ & $-1 \cdot 48 \%(-2 \cdot 8$ to $-0 \cdot 2 \%)$ & $0 \cdot 028$ \\
\hline Mean cost per patient $(€)$ & & $2155 \cdot 30 \pm 1620$ & $1911 \cdot 12 \pm 507 \cdot 23$ & $-243 \cdot 65(-365 \cdot 18$ to $-123 \cdot 18)$ & $0 \cdot 0001$ \\
\hline
\end{tabular}

* Groups have been defined according to individual DRG costs (Supplementary Table S3): I (1500-2200€); II (<1500€); III (>2200€).

$\dagger$ Each of these GRDs represented a median cost for the entire study period that was over $€ 2000$ per hospital admission.

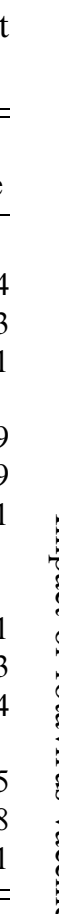


DRGs are universally recognized tools used to optimize Health Systems [22]. However, to date, we only found two DRG-based studies in Spain, investigating the cost-effectiveness for infectious diseases, both not dealing with vaccines [23, 33]. According to a national study, the introduction of universal vaccination with RotaTeq $^{\circledR}$ would not be cost-effective in Spain [34]. Notwithstanding, more research was needed on real cost savings. We only observed a significant cost decrease for more highly weighted DRGs accompanied by a significant decline in LOS of almost four days, despite the number of cases did not decrease (Table 4). On the contrary, the major descent in the number of hospitalizations occurred in group II, with a significant decrease in LOS by half a day, but with an increased cost. Regarding group I, the costs increased, but the mean stay did not change. Therefore, in the vaccination period, the less complicated hospitalizations due to rotavirus (group II) fell by $20 \%$ (from 604 to 481 ), at the cost of only an $11 \%$ increase (from 145 to 160 ) in hospitalizations of ARGEs with complications (group I). If unit mean costs owing to each I, II and III DRG groups had not increased so much in 2007-2009, overall savings we have estimated would have been even greater. It seems that a probable lower efficiency in the context of a greater complexity of the own hospitalization units for rotavirus (pediatrics and neonatology) over the years might have directly expanded the mean costs estimations by DRG in 2007-2009. That is because the increase in costs would have exceeded the increase in complexity. The hypothesis of a higher complexity in 2007-2009 is because the case mix (or casuistic) index (CIX) from pediatric and neonatology units of SESCAM indicates that the casuistry of the analyzed series in the vaccine period (CIX 0.58 ) was somewhat higher than that of the pre-vaccine period (CIX 0.37) (Supplementary Table S2). The supposition of a probable lower efficiency is because the performance or (functional) index of both 3-year periods reveals that those units of SESCAM attended its patients with an average mean stay similar to that of Spain; despite of its complexity was significantly lower than that of the SNHS (Supplementary Table S2).

The selling price of Rotateq ${ }^{\circledR}$ for complete immunization to the public in pharmacies at the end of the study period was about $€ 207$, while the selling price of the reference laboratory was $€ 133 \cdot 5$. In Spain, there is no option that the cost of vaccines not included in the universal national immunization program will be partially reimbursed by the Government. That is, the rotavirus vaccination is fully self-financed by consumers, which makes it difficult to acquire for families facing economic difficulties. The selling price of Rotateq ${ }^{\circledR}$ for complete immunization to the public in pharmacies at the end of the study period was about $€ 207$, while the selling price of the reference laboratory was $€ 133 \cdot 5$. Then, while savings of $€ 26071$ due to the decrease (by 107 cases) in rotavirus hospitalizations during the 2007-2009 period was a consequence of patients self-financing the vaccination at a cost of $€ 22149(107 * 207)$ for them, the vaccination cost for the SNHS would actually be lower, namely $€ 14285$ $(107 * 134)$. If the actual level of $34 \%$ coverage reached in 2007-2009 had been provided by a public immunization program at that lower per unit cost, the vaccine would have been cost-effective because the expense of the vaccination for the SNHS represents $€ 11786.05$ less than the overall savings we have estimated. Furthermore, a national immunization program would let reach a higher coverage and so, get more sustained saving over the time.

Long-term trends in Europe suggest an increase of campylobacteriosis $[35,36]$. We discovered a decrease by $28 \%$ in children under 5 years of age, and LOS and extended LOS for Campylobacter decreased significantly by almost $1 \frac{1}{2}$ days and by $68 \%$, respectively. One reason for this decrease could be simultaneous co-infection with rotavirus. Although viruses are the most commonly reported agents of gastrointestinal co-infections accompanying rotavirus [37, 38], Campylobacter spp. is the most frequent cause of enteropathogenic concomitant AGE for ARGEs, present in up to $19 \%$ of cases [38]. While co-occurrence of rotavirus and Campylobacter was coded in 5\% of hospitalizations in children under 5 years of age during 2003-2005, this co-infection was not present during 2007-2009. This would contribute to the decrease observed in stays for campylobacteriosis during 2007-2009, as co-infections involve an increased assistance during hospitalization [39]. Although very little is known about the role of interaction between pathogens in causing diarrhea, a recent study has reported that the co-occurrence of rotavirus and Campylobacter jejuni only occurs in symptomatic children $(P<0.0001)$ and that Salmonella usually does co-occur with norovirus better than with rotavirus [38].

Our study has some limitations. Although MBDS was designed for financial management purposes, certain incremental change in health costs over time is 
presumed to be inevitable. Despite we took into consideration the yearly Consumer Price Index, other factors influencing the incremental mean unit costs of DRGs over the years may have contributed to an underestimation of the real savings. The recognition of the health workers' Professional Career status that began in 2006 is a good example, which of course might have entailed an increase in the budget set to health personnel salaries. We also commented above a lower efficiency of pediatric and neonatology units, which means incremental costs exceeding an incremental complexity. Actually, there was an increase in public health expenditure of SESCAM despite the economic crisis that started in Spain in 2008 (Supplementary Fig. S1). There is also the possibility of underestimation in the recruitment of cases from the discharge database. The dependency of our estimations on accuracy coding remains a problem when comparing and interpreting trends in the five provinces of CLM, even though the variable "provinces' is a field always fulfilled in the MBDS. In this sense, we found some differences in the decrease in LOS and in the incidence rate by provinces [4], despite we found no province-specific differences in vaccine coverage $(P=0.599)$. Finally, it is well known that the true burden of community-acquired rotaviruses is generally misclassified when estimated in hospital databases through ICD-9-CM [8], with a maximum sensitivity of $47 \%$ [40]; albeit this underestimation that is supposed to affect equally both periods of comparison would not influence the estimation of changes occurring between them.

The biggest strength is that this research constitutes the first national study published using the DRG system to evaluate cost savings after a period of vaccine implementation. The 2007-2009 study period coincided with the above mentioned economic crisis, therefore some LOS descent findings may be expected to be due to economic constrains, more than the vaccines. But the decrease in rotavirus hospitalization costs gives meaning to the trend to a lower LOS that only became significant for rotavirus and Campylobacter (the last, the most frequent cause of enteropathogenic concomitant AGE for ARGEs), which would reflect a modest vaccine impact more than health policy changes or an improvement in the AGE management.

In summary, the actual modest decrease in hospital costs after the 3-year period of simultaneous Rotarix ${ }^{\circledR}$ and Rotateq ${ }^{\mathbb{R}}$ availability in Spain was due to cases with higher DRG weights; and the decrease in LOS would represent a faster recovery of such complex cases or even of more moderate-to-mild cases hospitalized post-introduction. Alongside this, the consequent savings in social costs according to previous studies might have been even fivefold higher than hospital savings; to which we would even have to add a specific saving in social costs derived from a lower parents' absenteeism, as a consequence of the average stay decrease in the vaccination period. Despite a universal rotavirus vaccination program has been discarded in Spain for not being cost-effective overall, the present study provides a first step toward re-evaluating its implementation, regarding its potential high impact on both at-risk children and societal costs.

\section{SUPPLEMENTARY MATERIAL}

The supplementary material for this article can be found at https://doi.org/10.1017/S0950268817000620

\section{ACKNOWLEDGEMENTS}

The authors would like to thank Rosario Valverde Vaquero and Inés Ruano del Cerro, from the Economic Management Control, General Secretary of the Health Service of Castile-La Mancha in Toledo, who provided disaggregated data on costs by years and hospitals, allowing us to perform exact estimations of costs. The authors have no financial relationships to disclose.

\section{DECLARATION OF INTEREST}

None.

\section{REFERENCES}

1. PROTECT. The paediatric burden of rotavirus disease in Europe. Epidemiology and Infection 2006; 134: 908-916.

2. Saia M, et al. Hospitalisation for rotavirus gastroenteritis in the paediatric population in the Veneto Region, Italy. BMC Public Health 2010; 10: 636.

3. Flem E, et al. Rotavirus gastroenteritis in Norway: analysis of prospective surveillance and hospital registry data. Scandinavian Journal of Infectious Diseases 2009; 41(10): 753-759.

4. Redondo O, Cano R, Simón L. Decline in rotavirus hospitalizations following the first three years of vaccination in Castile-La Mancha, Spain. Human Vaccines \& Immunotherapeutics 2015; 11(3): 769-775. doi: 10.1080/21645515.2015.1009339.

5. Le Saux N, et al. Substantial morbidity for hospitalized children with community-acquired rotavirus infections: 2005-2007 IMPACT surveillance in Canadian 
hospitals. The Pediatric Infectious Disease Journal 2010; 29(9): 879-882. doi: 10.1097/INF.0b013e3181e20c94.

6. Ogilvie I, et al. Burden of community-acquired and nosocomial rotavirus gastroenteritis in the pediatric population of Western Europe: a scoping review. BMC Infect Dis. 2012; 12: 62. doi: 10.1186/1471-2334-12-62.

7. Wildi-Runge $\mathbf{S}$, et al. A 4-year study on clinical characteristics of children hospitalized with rotavirus gastroenteritis. European Journal of Pediatrics 2009; 168(11): 1343-1348. doi: 10.1007/s00431-009-0934-z.

8. Mast TC, et al. Burden of childhood rotavirus disease on health systems in the United States. The Pediatric Infectious Disease Journal 2010; 29(2): e19-e25.

9. Marsella M, et al. Epidemiology of rotavirus-associated hospital admissions in the province of Ferrara, Italy. European Journal of Pediatrics 2009; 168(12): 14231427. doi: 10.1007/s00431-009-0942-z.

10. Giaquinto $\mathbf{C}$, et al. Costs of community-acquired pediatric rotavirus gastroenteritis in 7 European countries: the REVEAL Study. Journal of Infectious Diseases 2007; 195(Suppl. 1): S36-S44.

11. Diez-Domingo J, et al. Burden of paediatric rotavirus gastroenteritis (RVGE) and potential benefits of a universal Rotavirus vaccination programme with a pentavalent vaccine in Spain. BMC Public Health 2010; 10 (1): 469. doi: 10.1186/1471-2458-10-469.

12. Pockett RD, et al. A comparison of healthcare resource use for rotavirus and RSV between vulnerable children with co-morbidities and healthy children: a case control study. Journal of Medical Economics 2013; 16(4): 560565. doi: 10.3111/13696998.2013.774278.

13. Rowinsky PA, et al. Ancillary testing in children with rotavirus gastroenteritis. Journal of Hospital Medicine 2009; 4(7): E36-E40. doi: 10.1002/jhm.487.

14. Desai R, et al. All-cause gastroenteritis and rotaviruscoded hospitalizations among US children, 2000-2009. Clinical Infectious Diseases 2012; 55(4): e28-e34. doi: 10.1093/cid/cis443.

15. Kilgore A, et al. Rotavirus-associated hospitalization and emergency department costs and rotavirus vaccine program impact. Vaccine 2013; 31(38): 4164-4171. doi: 10.1016/j.vaccine.2013.06.085.

16. Paulke-Korinek M, et al. Sustained low hospitalization rates after four years of rotavirus mass vaccination in Austria. Vaccine 2013; 31(24): 2686-2691. doi: 10.1016/j.vaccine.2013.04.001.

17. Zlamy M, et al. The impact of rotavirus mass vaccination on hospitalization rates, nosocomial rotavirus gastroenteritis and secondary blood stream infections. BMC Infectious Diseases 2013; 13: 112. doi: 10.1186/ 1471-2334-13-112.

18. Karmann A, Jurack A, Lukas D. Recommendation of rotavirus vaccination and herd effect: a budget impact analysis based on German health insurance data. The European Journal of Health Economics 2015; 16(7): 719-731. doi: 10.1007/s10198-014-0624-2.

19. Gil-Prieto R, et al. Impact of non-routine rotavirus vaccination on hospitalizations for diarrhoea and rotavirus infections in Spain. Vaccine 2013; 31(43): 5000-5004. doi: 10.1016/j.vaccine.2013.05.109.
20. Dudareva-Vizule S, et al. Impact of rotavirus vaccination in regions with low and moderate vaccine uptake in Germany. Human Vaccines \& Immunotherapeutics 2012; 8(10): 1407-1415. doi: 10.4161/hv.21593.

21. Redondo-González $\mathbf{O}$. Validity and reliability of the minimum basic data set in estimating nosocomial acute gastroenteritis caused by rotavirus. Revista Española de Enfermedades Digestivas 2015; 107(3): 152-161.

22. Rivero A, et al. Analysis and Development of the GDRs in the National Health System. Madrid: Publications Center of Ministry of Health and Consumer Affairs, 1999.

23. Gómez $\mathbf{J}$, et al. Clinical and economic analysis of an internal medicine-infectious disease department at a university general hospital (2005-2006). Enfermedades Infecciosas y Microbiología Clínica 2009; 27(2): 70-74. doi: 10.1016/j.eimc.2008.05.001.

24. Palmieri F, et al. Early discharge of infectious disease patients: an opportunity or extra cost for the Italian Healthcare System? Le Infezioni in Medicina 2013; 21 (4): 270-278.

25. Kanerva M, Ollgren J, Lyytikäinen O. Finnish Prevalence Survey Study Group. Benchmarking antibiotic use in Finnish acute care hospitals using patient case-mix adjustment. Journal of Antimicrobial Chemotherapy 2011; 66(11): 2651-2654. doi: 10.1093/jac/dkr333.

26. Kanerva M, Ollgren J, Lyytikäinen O. Finnish Prevalence Survey Study Group. Interhospital differences and case-mix in a nationwide prevalence survey. The Journal of Hospital Infection 2010; 76(2): 135138. doi: 10.1016/j.jhin.2010.05.017.

27. Serrais J, Mallolas J, Ribas J. Direct pharmaceutical costs, diagnostic related groups and CD4 cell count in hospitalized HIV-infected patients. Medicina Clínica (Barc). 1997; 109(10): 361-363.

28. Mannocci A, et al. Epidemiology and direct medical costs of human leishmaniasis in Italy. Journal of Preventive Medicine and Hygiene 2007; 48(1): 27-36.

29. Redondo-González O, Tenías-Burillo JM. A multifactorial regression analysis of the features of community-acquired rotavirus requiring hospitalization in Spain as represented in the minimum basic data set. Epidemiology and Infection 2016; 144(12): 2509-2516. doi: $10.1017 / \mathrm{S} 0950268816000881$.

30. Bouzón-Alejandro M, et al. Prospective evaluation of indirect costs due to acute rotavirus gastroenteritis in Spain: the ROTACOST study. BMC Pediatrics 2011; 11: 81. doi: 10.1186/1471-2431-11-81.

31. Gil A, et al. Burden of hospitalizations attributable to rotavirus infection in children in Spain, period 1999 2000. Vaccine 2004; 22: 2221-2225.

32. Marocco A, et al. Hospitalisation associated with Rotavirus gastroenteritis in Italy, 2001-2003, evaluated by means of ICD9-CM diagnostic codes. Igiene e Sanità Pubblica 2006; 62(2): 215-244.

33. Arteaga-Rodríguez A, et al. Changes in the epidemiology of hepatitis A in Spain (2005-2008): trends of acute hepatitis A hospitalizations, comorbidities, and costs associated with the hospitalization. European Journal of Gastroenterology \& Hepatology 2010; 22 (11): 1284-1289. 
34. Imaz Iglesia I, et al. Análisis Coste-Utilidad de la Introducción de la Vacunación Universal frente al Rotavirus en España. Madrid: Agencia de Evaluación de Tecnologías Sanitarias - Ministerio de Ciencia e Investigación - Instituto de Salud Carlos III, 2011.

35. Schielke A, Rosner BM, Stark K. Epidemiology of campylobacteriosis in Germany - insights from 10 years of surveillance. BMC Infectious Diseases 2014; 14: 30. doi: 10.1186/1471-2334-14-30.

36. Maraki S, et al. Long-term trends in the epidemiology and resistance of childhood bacterial enteropathogens in Crete. European Journal of Clinical Microbiology \& Infectious Diseases 2012; 31: 1889-1894.

37. Wolak Z, Wałaszek MZ, Dobroś W. Incidence of healthcare-associated gastroenteritis in children hospitalized on paediatric ward in provincial hospital in 2007-2013. Przeglad Epidemiologiczny 2015; 69: 33-39.

38. Valentini D, et al. Coinfection in acute gastroenteritis predicts a more severe clinical course in children. European Journal of Clinical Microbiology \& Infectious Diseases 2013; 32(7): 909-915. doi: 10.1007/s10096-013-1825-9.

39. Li LL, et al. Aetiology of diarrhoeal disease and evaluation of viral-bacterial coinfection in children under 5 years old in China: a matched case-control study. Clinical Microbiology and Infection 2016; 22(4): 381. e9-381.e16. doi: 10.1016/j.cmi.2015.12.018.

40. Hsu VP, et al. Use of active surveillance to validate international classification of diseases code estimates of rotavirus hospitalizations in children. Pediatrics 2005; 115(1): 78-82. 